\title{
Alteridade e empatia: virtudes essenciais para a formação do cirurgião-dentista
}

\author{
Marcelo Mocelin Bark*; Marjory Posanski*; Kauhanna Vianna de Oliveira**; João Armando \\ Brancher***; Léo Kriger****; Marilisa Carneiro Leão Gabardo******
}

* Estudante de Graduação em Odontologia, Universidade Positivo

** MSc. Programa de Pós-Graduação em Odontologia, Universidade Positivo

*** PhD. Programa de Pós-Graduação em Odontologia, Universidade Positivo

**** MSc. Faculdade de Odontologia, Pontifícia Universidade Católica do Paraná

***** PhD. Programa de Pós-Graduação em Odontologia, Universidade Positivo

Recebido em 14/12/2017. Aprovado em 18/03/2018.

\begin{abstract}
RESUMO
$\mathrm{O}$ atendimento profissional vinculado à saúde se deve a certas premissas que ultrapassam o aspecto meramente técnico. Para que transformações ocorram, a atenção às mudanças curriculares é indispensável durante a formação do cirurgião-dentista. $\mathrm{O}$ ensino superior carece de estratégias que remodelem a forma de se pensar o outro. Empatia e alteridade são virtudes que, se desencadeadas, podem alterar a relação profissional-paciente. Buscou-se elucidar o significado desses conceitos, como proposta reflexiva em consonância com o atendimento humanizado em saúde, as Diretrizes Curriculares Nacionais (DCN), e o contato com o Sistema Único de Saúde (SUS), como propulsores de transformações no meio acadêmico. Adotou-se a revisão narrativa da literatura, pela pesquisa documental, contemplando a legislação nacional acerca da educação superior em Odontologia, somada a consulta às bases científicas Medline, LILACS e SciELO. As palavras-chave empregadas foram: alteridade, empatia, empathy, humanização da assistência e humanization of assistance, no período de 2004 a 2017. Foram selecionados textos completos na área odontológica que contemplassem a interrelação entre os temas. A síntese qualitativa da literatura consultada permitiu a observação de que houve uma mudança, ainda não totalmente concretizada, mas relevante, com relação à ultrapassagem do foco apenas técnico e curativo, como bem proposto nas DCN e tão almejado pelo SUS quanto ao profissional ingressante na vida pública. Concluiu-se que o atendimento humanizado em Odontologia ainda é um desafio. Contudo, mudanças acadêmicas que suscitem o despertar das virtudes de alteridade e empatia devem se concretizar.
\end{abstract}

Descritores: Virtudes. Educação Superior. Sistema Único de Saúde. 


\section{INTRODUÇÃ̃O}

Tendo em vista que somente o tecnicismo procedimental não esgota o ideal de uma abordagem de excelência, a assistência multiprofissional à saúde se torna um desafio para os profissionais do ramo ${ }^{1}$. Desafio que instiga o readequar do trato, fazendo emergir a necessidade de uma visão holística sobre o ser humano, ou seja, uma abordagem integral, que envolva aspectos biológicos, psicológicos e sociais $^{1}$.

Com vistas à formação do cirurgiãodentista com visão ampliada, as Diretrizes Curriculares Nacionais (DCN) para o curso de graduação em Odontologia orientam sobre o perfil "ideal" do egresso/formando ${ }^{2}$. O documento apregoa características generalistas, humanas, críticas e reflexivas, que devem pautar a atuação do profissional da saúde em qualquer nível. Também, aponta para que a base de ação procedimental siga os rigores técnicos e científicos com base em princípios éticos, legais e pela compreensão referente às realidades sociais, culturais e econômicas ${ }^{2}$.

De modo a abranger todos os aspectos supracitados, resgata-se a importância do desenvolvimento das relações interpessoais ${ }^{3}$. $\mathrm{O}$ primordial está em se pensar no paciente como um "ser", não esgotando ao profissional somente a preocupação com sinais e sintomas ${ }^{3}$. A humanização do atendimento em Odontologia requer mais do que intelectualidade acadêmica, mas também acolhimento e sinceridade relacional, criando e desenvolvendo características comportamentais, ou seja, virtudes. Os profissionais da área da saúde devem reconhecer a Política Nacional de Humanização ${ }^{4}$, para que cuidados de qualidade sejam dados e para que se promovam a reunião, o acolhimento e o reconhecimento de si mesmo ${ }^{5}$.

Com isso, as virtudes comportamentais aplicadas a outros podem trazer como recompensa o próprio bem-estar ${ }^{6}$. Então, requerse a reflexão sobre o bem-estar subjetivo, ou seja, satisfação e gratidão.

Mediante o exposto, este trabalho teve como objetivo revisar, de modo narrativo, os conceitos de empatia e de alteridade, como proposta reflexiva acerca dessas virtudes e o atendimento humanizado em saúde, de modo a revelar características comportamentais necessárias à formação acadêmica em Odontologia, aliado ao debate das propostas das DCN, e o contato com o Sistema Único de Saúde (SUS), como propulsores de transformações no meio acadêmico.

\section{PERCURSO METODOLÓGICO}

A revisão narrativa buscou fundamentar e contextualizar as virtudes de empatia e alteridade junto aos preceitos da humanização do atendimento em saúde, à formação proposta nas DCN, com reflexos no SUS.

O levantamento bibliográfico foi realizado inicialmente por pesquisa em livros-texto específicos e documentos da legislação nacional que contemplassem o ensino superior em Odontologia. As bases de dados consultadas foram National Library of Medicine (PubMed/NLM), Literatura Latino-Americana em Ciências da Saúde (LILACS) e Scientific Eletronic Library Online (SciELO), utilizando as palavras-chave: alteridade, empatia, empathy, humanização da assistência e humanization of assistance. Esses descritores foram oriundos de artigos previamente publicados, bem como dos Descritores em Ciências da Saúde (DeCS) e Medical Subject Headings (MeSH). O período de busca compreendido foi de 2004 a 2017.

Adotou-se a seleção de textos completos em português, inglês ou espanhol. Não foram incluídos editoriais, teses e dissertações. Os resumos ou abstracts foram lidos para que o conteúdo fosse analisado, de modo que fossem 
selecionados aqueles relacionados com o escopo central desse estudo. Em seguida, deu-se incío à leitura dos textos na íntegra, para agrupá-los em categorias temáticas.

\section{RESULTADOS}

\section{Ética, Educação e Acolhimento}

Ao se pensar em Educação, não se pode apenas trazer à memória a boa formação escolar e, possivelmente, a universitária. O tema está intimamente ligado à reflexão sobre a melhor maneira de viver. A busca da identificação dessa melhor forma de viver e conviver, essencialmente, guarda relação com a Ética. Essa, não pode ser entendida de modo pragmático como comportamentos adequados ou não adequados à sociedade ${ }^{7}$. Os autores ainda citam que "Ética tem a ver com convivência. Eis seu objeto. Mas seu entendimento e compreensão implicam também esforço intelectual porque é pensamento sobre a vida partilhada, sobre as relações. É um saber que mobiliza, que vem pelo outro e que curiosamente está ausente da nossa educação formal. Na escola, a reflexão sobre a convivência ocupa posição marginal ou nula"”.

Entendendo que a busca pela melhor forma de viver e conviver é, indispen-savelmente, favorecida pela vida partilhada e suas relações interpessoais, nota-se a relevância do acolhimento humano quando se delibera sobre o atendimento odontológico de qualidade.

Com referência ao acolhimento, este se trata de ouvir aqueles que procuram atendimento, e assim dar respostas mais adequadas, o que favorece os processos de trabalho em saúde. Trata-se da oferta de um atendimento orientado, responsável e resolutivo $^{8}$. Para Hennington ${ }^{9}$, acolher significa humanizar o atendimento.

Dentro da Política Nacional de Humanização do SUS, o acolhimento, enquanto diretriz, em sua faceta ética "se refere ao compromisso com o reconhecimento do outro, na atitude de acolhê-lo em suas diferenças, suas dores, suas alegrias, seus modos de viver, sentir e estar na vida" .

Acadêmicos e profissionais da área odontológica vivenciam diariamente experiências relatadas por pacientes, logo, não só patologias e desconfortos relacionados à cavidade bucal são expressos, mas, não raras vezes, as condições da vida conjugal, em família, no trabalho, bem como, ansiedades e angústias das rotinas. $\mathrm{O}$ atendimento com base na abordagem integral ao ser humano, inclusive previsto nas $\mathrm{DCN}^{2}$, requer vínculo, acolhimento e, sobretudo, compreensão e entendimento das mudanças. Então, percebe-se que "Para lidar com a mudança, você precisa, sobretudo, prestar atenção nas pessoas. Paulo Freire, o brasileiro que mais acumulou títulos de doutor honoris causa na história do nosso país, também era um mestre nisso. Quando alguém vinha falar com ele, um homem mundialmente famoso, Paulo não só parava para escutar como dava toda a atenção do mundo. Não raro, colocava a mão no ombro do interlocutor para criar uma condição de igualdade, um vínculo, uma conexão física para materializar o que Aristóteles chamou de amizade: dois corpos numa única alma"10.

Contudo, uma análise crítica permite a identificação dos desafios enfrentados quanto ao acolhimento nas práticas em saúde ${ }^{11}$, inclusive as bucais $^{12,13}$. No que tange ao aspecto profissional, existe uma falta de compreensão do real significado do acolher ${ }^{12,13}$.

\section{Empatia e Alteridade}

Trazer a debate o conceito de humanização no atendimento odontológico evoca percepções importantes quanto à evolução técnica e científica em Odontologia, uma vez que tal área da saúde foi marcada pela desarmonia entre o tecnicismo e a qualidade de contato humano ${ }^{14,15}$. Evidencia-se a importância do acolhimento do paciente que sofre, e assim é requerida a 
transcendência dos conhecimentos relacionados à saúde bucal, de modo a entender que o processo de cura ultrapassa a área do saber científico ${ }^{14}$. Por tal, a profissão odontológica deve contemplar a habilidade técnica, a formação científica e o olhar humano da promoção da saúde ${ }^{16}$.

Uma das características comportamentais que contém a essência da boa interpessoalidade é a empatia. O estado de empatia denota a percepção correta sobre referências e internalizações do outro, de forma a se colocar no lugar de outrem, compreendendo os pensamentos e emoções evidenciados mediante alguma situação ${ }^{17}$.

A empatia é uma virtude útil, que também propicia vínculo, desencadeando uma "ponte afetiva" entre o profissional da saúde e o paciente $^{18}$. Assim, pelo caráter facilitador de vínculo, a empatia favorece a legitimação da humanidade, ou seja, a sensação de pertencimento é favorecida e aflora o sentimento de acolhimento, passando o indivíduo que demanda tratamento, a se sentir mais tranquilo em expor suas necessidades ${ }^{17}$. Aqui é possível ser feita uma reflexão acerca da importância da empatia durante a anamnese. É notório que o bom planejamento cirúrgico/terapêutico depende da qualidade e da especificidade das informações obtidas durante a mesma. A empatia pode ser entendida aqui como uma ferramenta auxiliar na coleta de informações pessoais necessárias ao plano de tratamento. É preferível revelar intimidades e informações aos que nos compreendem e acolhem ou aos cingidos de etiquetas que emanam o desinteresse das condições particulares?

Agindo de forma sinérgica à empatia, ambas almejando o vínculo interpessoal, a alteridade é a capacidade de enxergar o outro como um ser diferente, em outras palavras, aceitar a condição da existência de outras opiniões, culturas, saberes, aparências e crenças, sabendo que tal virtude não se enquadra em explicações generalizantes e inflexíveis ${ }^{19}$. Assim, nota-se a fundamental utilização da alteridade no processo de formação acadêmica, bem como na relação profissional-paciente.

Com base na perspectiva da alteridade, a educação é vista como uma construção evidenciada pela relação particular entre sujeitos de pensamentos distintos. Com isso, em meio ao processo de interação, não apenas a aprendizagem de conceitos e informações são obtidos, mas, sobretudo, o discernimento dos contextos que promovem a assimilação dos elementos de implacável significado ${ }^{19}$.

Especificamente no contexto das DCN, podem ser dados alguns exemplos de como essas virtudes podem auxiliar no desenvolvimento de competências como a atenção à saúde, uma vez que a responsabilidade não se encerra com o ato técnico, mas na resolução do problema de saúde; na tomada de decisões, uma vez que as condutas mais adequadas são desejadas. Além disso, estão diretamente relacionadas às competências que envolvem de modo direto ou não, a comunicação e a liderança.

\section{Atendimento Humanizado em Odontologia}

Internalizados os significados da empatia e alteridade, evidencia-se a proposta da humanização no atendimento em Odontologia. $\mathrm{O}$ conceito de humanização é entendido como ação afável e tratável, ou seja, o tratamento benévolo e bondoso para com o outro ${ }^{20}$. Todavia, é evidente que entre a teoria e a prática da humanização no atendimento há um abismo, visto que essa metodologia de ensinoaprendizagem requer mudanças no que se referem às atitudes, comportamentos, valores, culturas, conceitos, dentre outros fatores ${ }^{16,20}$.

Desta forma, construir projetos pedagógicos humanizados é um desafio para as instituições de ensino superior $(\text { IES })^{21}$. 
Humanizar o atendimento não se limita a apenas chamar o paciente pelo nome e possuir constantemente um sorriso nos lábios, mas contemplar o entendimento dos medos, angústias e incertezas expostas pelo mesmo e com isso, proporcionar apoio e atenção permanente ${ }^{22}$.

Com base nos preceitos expostos na Resolução CNE/CES $3^{2}$, referente à uma formação profissional que transborde as tecnicalidades, o Ministério da Saúde e da Educação, em parceria, implementaram em algumas IES o Programa Nacional de Reorientação da Formação Profissional em Saúde (Pró-Saúde) ${ }^{20}$. A fim de abranger as novas tendências da formação acadêmica, o Pró-Saúde lança uma oportunidade acadêmica-educacional de desenvolvimento de habilidades relacionadas ao atendimento humanizado, inserindo o aluno na rede do SUS desde o início da formação acadêmica $^{20}$. Estudos apontam que esses Programas ampliam o desenvolvimento da educação interprofissional ${ }^{23,24}$.

\section{Inserção no SUS x Dificuldades}

Uma forma de proporcionar a atuação profissional esperada, a saber, interdisciplinar e integralizada, é o contato com o SUS, podendose assim desenvolver habilidades pessoais que favoreçam à saúde, como descritas nas $\mathrm{DCN}^{2,25}$. Os cursos de Odontologia carecem de uma readequação de seus sistemas de formação, para que se contribua para a capacitação de profissionais que atendam às demandas do sistema ${ }^{16,21,25}$. Com base nessas, nota-se que os procedimentos de mudança geram esperança quanto às novas possibilidades de iniciativa que possam favorecer o maior contato dos discentes com a prática profissional vinculada à Saúde Coletiva $^{26}$.

Todavia, não se pode negar a real presença de dificuldades. São as intempéries relacionadas à inserção do cirurgião-dentista no SUS: dificuldade da percepção social das práticas de saúde e do alcance das intervenções; conceito de saúde-doença limitado, dada a não incorporação da dimensão social; baixa efetividade das ações de promoção de saúde desencadeada pela não participação social; baixa incorporação das diretrizes propostas pelo SUS; saúde bucal fortemente pautada pelo modelo clínico; isolamento, ou seja, não interdisciplinaridade; e privilégio das ações curativas ${ }^{25}$. Mas, para que as dificuldades sejam superadas, a reorganização da matriz curricular, bem como o desenvolvimento de propostas que atendam aos requisitos da realidade do sistema público de saúde, são indispensáveis. De acordo com tais necessidades, o Conselho Federal de Educação ${ }^{26}$ propõe a modernização dos currículos dos cursos de graduação, o que deixa evidente a importância da formação profissional para a realidade do país, do perfil generalista capaz de aplicar a filosofia preventiva e social à saúde, da valorização e compreensão da participação social, da abordagem intersetorial e da prática extramuros sob supervisão em instituições públicas ${ }^{26}$.

\section{Multiprofissionalismo x Tecnicismo}

Ao longo do desenvolvimento dessa revisão de literatura, evidenciou-se a teoria do que seria uma formação/atuação/capacitação completa de um profissional cirurgião-dentista ${ }^{2}$, bem como, alguns meios e mudanças acadêmicas necessários para que as virtudes fundamentais, empatia e alteridade, possam fazer surgir o que se espera de um atendimento de qualidade, a saber, a humanização do atendimento em saúde.

A proposta é para que sejam desenvolvidas habilidades que não promovam isolamento das realidades do dia a dia, mas proporcionem o enfrentamento de todo tipo de dificuldade. A inserção na realidade necessitada, ou seja, no sistema público de saúde exige convivência com a pluralidade comportamental. 
A compreensão mecanicista acachapa a consciência e a torna um mero reflexo das coisas materiais ${ }^{27}$. Portanto, emerge a necessidade do entendimento das subjetividades para que a consciência seja hipertrofiada ${ }^{27}$. É notável o potencial produtivo que a interação da graduação em Odontologia com o SUS pode desencadear. É nesse contato com a realidade do país que o educar, ensinar e tratar vai sendo construído com a consciência dos verdadeiros valores para vida, pois educar exige pesquisa, exige respeito aos saberes do educando, reflexão crítica sobre a prática, bom senso, apreensão da realidade, alegria e esperança, convicção de que a mudança é possível, saber escutar, e exige, sobretudo, a tomada consciente de decisões ${ }^{27}$.

Mediante o exposto, o trabalho intelectivo deve transbordar as inovações teóricas e se aplicar nas práticas acadêmicas dos futuros profissionais da área da saúde. Também, tal prática voltada à formação generalista, deve ter seu conteúdo internalizado por docentes, a fim de romper com os limites da própria formação, que muitas vezes se encontra enlaçada pelos conceitos tecnicistas ${ }^{28}$.

Revela-se necessária a luta contra os "nós críticos" presentes na formação profissional. O termo "nó crítico" se refere ao modelo de ensino equivocado e que colide com a busca de egressos que possuam currículo e experiência que contemple as necessidades sociais da saúde, ou seja, a realidade do SUS. A ênfase tecnicista, individualizada e "medicalizada" demonstra o modelo de ensino fragmentado e que se apresenta como um verdadeiro "nó" impeditivo da reforma curricular que busca o atendimento humanizado ${ }^{28}$.

A ética, sendo entendida como busca intelectual para melhora da convivência e compartilhamento de experiências, traduz-se como prática de vida ${ }^{7}$. Pode-se visualizar que as DCN para graduação em Odontologia são chave fundamental para a formação nesse campo, ou seja, em harmonia com os requisitos essenciais que um cirurgião-dentista deve ter ${ }^{2}$.

Embora tenham ocorrido inovações reflexivas acerca das novas abordagens curriculares para profissionais da área da saúde, a Odontologia se evidenciou atrasada historicamente quanto aos requisitos ${ }^{29}$. Mais um "nó" a se desatar, que objetiva a conformação prática ao modelo multiprofissional $\mathrm{e}$ interdisciplinar proposto pelas $\mathrm{DCN}^{2}$. A implementação das DCN buscou a reformulação das matrizes curriculares e, consequentemente, a atualização das capacidades discentes, dentre as quais: o pensar de forma crítica, a capacidade de tomar decisões, a atuação em equipes multiprofissionais, o planejar estrategicamente para contínuas mudanças, o administrar e gerenciar serviços de saúde e a perenidade do aprendizado $^{29}$. Então, ressalta-se a importância da inserção do discente no SUS desde o início de sua formação acadêmica ${ }^{20}$, evidenciando a indissociável relação entre educação e saúde, onde há uma dependência evolutiva entre SUS, DCN e formação acadêmica ${ }^{29}$.

Morita e Kriger $^{29}$ explanam que atuar de forma multiprofissional demanda um romper do trabalho centrado na individualidade, buscando o desenvolver do trabalho em equipe, e então emergem compartilhamentos, planejamentos, tarefas e se estabelece a cooperação e colaboração. As equipes interdisciplinares e transdisciplinares estão associadas ao aumento da qualidade dos cuidados ${ }^{30}$.

Transbordando a limitante compreensão mecanicista $^{27}$ e arvorando o comportamento profissional generalista ${ }^{2,22}$, evidenciaram a empatia e a alteridade como fundamentos para o florescer da humanização do atendimento em Odontologia. Destacou-se a inclusão do discente, desde os primeiros anos acadêmicos, na realidade do $\mathrm{SUS}^{20}$, pois as modificações necessárias em 
prol do perfil profissional de excelência, requerem uma não dissociação entre teoria e prática, a saber $^{29}$. Dessa forma, por meio da compreensão e da absorção de tais valores e ferramentas, a individualidade no atendimento vai dando lugar à abordagem multiprofissional, à medida que o acadêmico interage com a realidade do SUS e, consequentemente, torna prática os conceitos de interdisciplinariedade e transdiciplinariedade ${ }^{29}$, ocasionando, gradativamente, o desatar dos "nós críticos" que se apresentam como desafios a serem superados $^{28}$.

Não obstante, é fundamental que o SUS tenha caráter interlocutor entre escolas na formulação e aplicação de projetos pedagógicos, não sendo apenas um campo de estágio ou afim prático $^{29}$. Ressalta-se que "é na compreensão do conceito de saúde, na possibilidade de se formar um profissional capaz de fazer uma contribuição permanente para a produção da saúde da sociedade em que vive que as Diretrizes se amplificam"29. Portanto, para que tal processo avance deve haver uma constante interação entre políticas de Educação e de Saúde, tendo em vista que a análise crítica das DCN remetem à reflexão de que profundas mudanças devem ocorrer para que os futuros profissionais consigam atender à demanda do SUS, ou seja, à realidade ${ }^{29}$.

Morita e Kriger $^{29}$ afirmam que o modelo pedagógico tradicional é ineficaz, pois não açambarca em seus conteúdos conceitos vinculados à realidade e às necessidades sociais. Com isso, o ideal didático-pedagógico que se almeja requer que o método de aprendizagem centrado no professor deva ser transformado em uma prática de múltiplos cenários que se adeque à realidade social. Com relação à articulação de conhecimentos, deve-se buscar a troca das experiências teórico-práticas em disciplinas isoladas por conteúdos e experiências de especialidades integradas. Já em relação ao trabalho multiprofissional, tal deve ocorrer entre profissionais de uma mesma disciplina, formando equipes de cada área da saúde. O quarto ponto fundamental se refere às transformações das práticas pedagógicas e faz referência aos cenários de aprendizagem. Deve ocorrer uma reformulação das clínicas de especialidades, de modo que, desde o início do curso, os alunos sejam inseridos em ambientes clínicos integrados. Portanto, a interação com o SUS desde o início da formação acadêmica favorece as mudanças almejadas e a construção de uma consciência multiprofissional. Experiências exitosas têm sido relatadas na literatura, sendo reiteradas as repercussões positivas no processo formativo $^{31-33}$

\section{CONSIDERAÇÕES FINAIS}

Não se espera que essas virtudes sejam utilizadas de forma pragmática, apenas objetivando a humanização do atendimento e, consequentemente, não as fornecendo de modo real ao profissional. Além disso, a humanização requer compromisso com a sinceridade, objetivando a melhor abordagem possível a um ser humano que necessita de ajuda.

Dada a importância do tema, bem como a incessante discussão sobre a melhor maneira de conviver, é inexorável um readequar dos objetivos profissionais quando nos referimos à saúde. Para que as virtudes de empatia e alteridade causem uma revolução, de modo a humanizar os atendimentos odontológicos, necessita-se de oportunidades em que a convivência com a realidade transforme consciências. Mais do que excelência nos procedimentos, o que também é indispensável, requer-se boa vontade e acolhimento no contato humano. Toda mudança é possível e as DCN, suportadas pelo SUS, têm esse propósito.

\section{ABSTRACT \\ Alterity and empathy: essential virtues for the formation of the dentist}

Professional care related to health must be based 
on certain assumptions that go beyond the technical aspect. For transformations to take place, attention to curricular changes is indispensable during the formation of the dental surgeon. The higher education lacks strategies that reshape the way one thinks about the other. Empathy and alterity are virtues that are unleashed, can alter the professional-patient relationship. The aim was to discuss the proposals of the National Curricular Guidelines (NCG), and the contact with the Unified Health System (UHS), as drivers of changes in the academic environment. It was adopted the narrative review of the literature, through documentary research, contemplating the national legislation on higher education in Dentistry, in addition to consulting the scientific bases Medline, LILACS, and SciELO. The keywords used were: alteridade, empatia, empathy, humanização da assistência e humanization of assistance, from 2004 to 2017. Full texts were selected in the dental area that contemplated the interrelationship between the themes. The qualitative synthesis of the literature consulted allowed the observation that there was a change, not yet fully realized, but relevant, in relation to the surpassing of the only technical and curative focus, as well proposed in the NCG and so longed for by the UHS as the professional in public life. Humanized care in Dentistry is still a challenge. However, academic changes that arouse the awakening of the virtues of alterity and empathy must materialize.

Descriptors: Virtues. Education, Higher. Unified Health System.

\section{REFERÊNCIAS}

1. Geniole LAI, Kodjaoglanian VL, Vieira CCA, Costa KC da. Assistência multidisciplinar à saúde: pós-graduação em assistência básica em saúde da família. Campo Grande: Editora UFMS: Fiocruz unidade Cerrado Pantanal; 2011.

2. Brasil. Conselho Nacional de Educação. Câmara de Educação Superior. Resolução CNE/CES 3. Institui Diretrizes Curriculares
Nacionais do Curso de Graduação em Odontologia (Fev 19, 2002).

3. Mota LQ, Farias DBLM, Santos TA dos. Humanização no atendimento odontológico: acolhimento da subjetividade dos pacientes atendidos por alunos de graduação em Odontologia. Arq Odontol. 2012; 48(3): 151-8.

4. Brasil. Ministério da Saúde. Secretaria de Atenção à Saúde. Núcleo Técnico da Política de Humanização. Humaniza SUS: documento base para gestores e trabalhadores do SUS. Brasília: Ministério da Saúde; 2010.

5. Silva RM, de Oliveira DC, Pereira ER. The discursive production of professionals about humanizing health: singularity, rights and ethics. Rev Latioam Enferm. 2015; 23(5): 936-44.

6. Siqueira MMM, Padovam VAR. Bases teóricas de bem-estar subjetivo, bem-estar psicológico e bem-estar no trabalho. Psicol Teor Pesqui. 2008; 24(2): 201-9.

7. Barros Filho $\mathrm{C}$ de, Pompeu J. A Filosofia explica as grandes questões da humanidade: Ética para uma vida boa. Rio de Janeiro: Casa da Palavra; 2013.

8. Brasil. Ministério da Saúde. Secretaria de Atenção à Saúde. Núcleo Técnico da Política Nacional de Humanização. Acolhimento nas práticas de produção de saúde / Ministério da Saúde, Secretaria de Atenção à Saúde, Núcleo Técnico da Política Nacional de Humanização. Brasília: Ministério da Saúde; 2010.

9. Hennington EA. Acolhimento como prática interdisciplinar num programa de extensão universitária. Cad Saúde Pública. 2005; 21(1): 256-65.

10. Cortella MS. O que a vida me ensinou: viver em paz para morrer em paz (paixão, sentido e felicidade). 5.ed. São Paulo: Saraiva; 2012. 
11. Neves M, Pretto SM, Ely HC. Percepções de usuários e trabalhadores de saúde sobre a implantação do acolhimento em uma unidade de saúde em Porto Alegre-RS, Brasil. Rev Odontol UNESP. 2013; 42(5):364-71.

12. Santos AM, Assis MMA, Oliveira AAAO, Nascimento MAA, Jorge MSB. Linhas de tensões no processo de acolhimento das equipes de saúde bucal do Programa Saúde da Família: o caso de Alagoinhas, Bahia, Brasil. Cad Saúde Pública. 2007; 23(1):7585.

13. Carnut L, Frazão MP, Moura Neto JG. Silva ALAS. Principais desafios do acolhimento na prática da atenção à saúde bucal: subsídios iniciais para uma crítica. Rev ABENO. 2017; 17(4):46-62.

14. Guerra CN, Bertoz APM, Fajardo RS, Alves Rezende MCR. Reflexões sobre o conceito de atendimento humanizado em Odontologia. Arch Health Invest. 2014; 3(6): 31-6.

15. Nascimento AC, Moysés ST, Bisinelli JC, Moysés SJ. Oral health in the Family health strategy: a change of practices or semantics diversionism. Rev Saude Publica. 2009; 43(3): 455-62.

16. Moysés SJ. Políticas de Saúde e formação de recursos humanos em Odontologia: os cursos de Odontologia devem avançar para um projeto pedagógico construído coletivamente, centrado no aluno como sujeito da aprendizagem e no professor como mediador do processo ensinoaprendizagem. Rev ABENO. 2004; 4(1): p. 30-7.

17. Tassinari MA, Durange WT. Experiência empática: da neurociência à espiritualidade. Rev Abordagem Gestalt. 2014; 20(1): 53-60.

18. Sueiras P, Romano-Betech V, VergilSalgado A, de Hoyos A, Quintana-Vargas S,
Ruddick W, et al. Today's medical self and the other: challenges and evolving solutions for enhanced humanization and quality of care. PLoS One. 2017; 12(7): e0181514.

19. Molar JO. Alteridade: uma noção em construção. Rev NUPEM. 2011; 3(5): 61-72.

20. Canalli CSE, Gonçalves SS, Chevitarese L, Silveira RG; Miasato JM. A humanização na Odontologia: uma reflexão sobre a prática educativa. Rev Bras Odontol. 2011; 68(1): 44-8.

21. Moysés SJ. A humanização da educação em Odontologia. Pro-Posições/UNICAMP. 2003; 14(1): 87-106.

22. Scalioni FAR, Alves RT, Mattos CLB de, Ribeiro RA. Humanização na Odontologia: a experiência da disciplina Odontopediatria II do Curso de Graduação em Odontologia da UFJF. Pesqui Bras Odontopediatria Clín Integr. 2008; 8(2):185-90.

23. Batista SHSS, Jansen B, Assis EQ, Senna MIB, Cury GC. Education in Health: reflections from the Pro-Health and PETHealth Programs. Interface (Botucatu). 2015; 19 Supl: 743-52.

24. Costa MV, Patrício KP, Câmara AMCS, Azevedo GD, Batista SHSS. Pro-Health and PET-Health as interprofessional education spaces. Interface (Botucatu). 2015; 19 Supl 1: 709-20.

25. Aerts D, Abegg C, Cesa K. O papel do cirurgião-dentista no Sistema Único de Saúde. Ciênc Saúde Coletiva. 2004; 9(1): 131-8

26. Palmier AC, Amaral JHL do, Werneck MAF, Senna MIB, Lucas SD. Inserção do aluno de Odontologia no SUS: contribuições do Pró-Saúde. Rev Bras Educ Méd. 2012; 36 (1 Supl. 2): 152-7.

27. Freire P. Pedagogia da autonomia: saberes necessários à prática educativa. São Paulo: Paz e Terra; 1996. 
28. Rossoni E， Lampert J. Formação de profissionais para o Sistema Único de Saúde e as Diretrizes Curriculares. Bol Saúde. 2004; 18(1): 87-98.

29. Morita MC, Kriger L. Mudanças nos cursos de Odontologia e a interação com o SUS: o conceito de saúde explicitado na Constituição e os princípios que nortearam a criação e implantação do SUS são fundamentais na definição das Diretrizes Curriculares dos cursos da área de Saúde. Rev ABENO. 2004; 4(1): 17-21.

30. da Silva RN, de Freitas FD, de Araújo FP, Ferreira MA. A policy analysis of teamwork as a proposal for healthcare humanization: implications for nursing. Int Nurs Rev. 2016; 63(4): 572-9.

31. Lucas SD, Palmier AC, Amaral João HL do, Werneck MAF, Senna MIB. Inserção do aluno de odontologia no SUS: contribuições do Pró-Saúde. Rev ABENO. 2011; 11(1): 29-34.

32. Biscarde DGS, Pereira-Santos M, Silva LB. Formação em saúde, extensão universitária e Sistema Único de Saúde (SUS): conexões necessárias entre conhecimento e intervenção centradas na realidade e repercussões no processo formativo. Interface (Botucatu). 2014; 18(48): 177-86.

33. Rocha NB da, Silva MC da, Silva IRG da, Lolli LF, Fujimaki M, Alves RN. Percepções de aprendizagem sobre disciplina interprofissional em Odontologia. Rev ABENO. 2017; 17(3): 41-54.

\section{Correspondência para:}

Marilisa Carneiro Leão Gabardo

e-mail: marilisagabardo@gmail.com

Rua Prof. Pedro Viriato Parigot de Souza, 5300 81280-330 Curitiba, PR 